\title{
Caracterización morfoestructural de tres poblaciones de Ovino de Pelo Criollo Colombiano "OPC"
}

Flórez, J.M.@; Hernández, M. de J.; Bustamante, M. de J. y Vergara, O.D.

Grupo de Investigación en Producción Animal Tropical. Universidad de Córdoba. Montería. Colombia.

Palabras ClaVe adicionales

Análisis multivariante.

Morfología.

Etiope.

Etnozootecnia.

Sudán.

\section{RESUMEN}

En los Ovinos de Pelo Criollos Colombianos (OPC) se distinguen principalmente los Sudán y Etiope, aunque no existe consenso en cuanto a su clasificación racial, puesto a que se carecen de estudios etnológicos que ayuden a sustentar si se deben considerar como una o más de una raza; por lo tanto, se tuvo como objetivo evaluar la variabilidad morfoestructural de ovinos Sudán y Etiope. Esta investigación fue desarrollada la región Caribe de Colombia. Fueron evaluadas un total de 180 ovejas Etiope, Sudán Bayo y Sudán Blanco. El análisis estadístico consistió en la estimación de estadística descriptiva, análisis de varianza $(P \leq 0,05)$, análisis de componentes principales, análisis discriminante canónico y correlaciones de Pearson. Para el análisis de los datos se utilizaron los softwares InfoStat $(20161)$ y SPSS $(19,0)$. En los resultados, se determinó que las Etiope poseen un menor potencial morfoestructural que las Sudán, dicha diferencia es estadísticamente significativa. Se requirieron sólo dos componentes principales para explicar el 100\% de la variabilidad de los datos. Las funciones canónicas estimadas presentaron la capacidad de separar significativamente a los OPC. Además, se determinó que el grado de homogeneidad en las ovejas evaluadas no fue bajo. A partir de este trabajo, se sugiere desde la morfología que los Sudán y Etiope sean catalogados como diferentes racialmente.

\section{Morphostructural characterization of three Colombian woolless sheep populations "OPC"}

\section{SUMMARY}

\section{ADDITIONAL KEYWORDS}

Multivariate analysis.

Morphology.

Etiope.

Ethnozootechny.

Sudan.

\section{INFORMATION}

Cronología del artículo.

Recibido/Received: 17.11.2016

Aceptado/Accepted: 22.04.2018

On-line: 15.07.2018

Correspondencia a los autores/Contact e-mail:

juliomateo7@hotmail.com

\section{INTRODUCCIÓN}

En la actualidad se reconocen diversas razas criollas de ovinos en Latinoamérica (DAD-IS, 2016). Sin embargo, Delgado et al. (2009) consideran que una parte de estas razas no se han caracterizado, clasificándose bajo el término "Criollos" a un gran número de razas perfectamente definidas y adaptadas a distintas condiciones ambientales.
Among Colombian woolless sheep (OPC) are mainly distinguished Sudán and Etiope sheep; however, there is no consensus regarding their breed classification due to a lack of ethnological studies which help to decide how they should be classified, as one or two breeds. The objective of this research was to evaluate the morphostructural variability of Sudán and Etiope sheep. This research was developed in the Caribbean region of Colombia. A total of 180 Etiope, Sudán Bayo and Sudán Blanco sheep were evaluated. The statistical analysis consisted in the estimation of descriptive statistics, analysis of variance $(\mathrm{P} \leq 0,05)$, principal components analysis, canonical discriminant analysis and Pearson correlations. For the analysis of the data, the InfoStat (20161) and SPSS (19.0) softwares were used. Results determined that Etiope sheep have a lower morphostructural potential than Sudán sheep. This difference is statistically significant. Two principal components were required to explain 100\% of the data variability. The estimated canonical functions presented the ability to significantly separate the OPC. Besides, it was determined that the degree of homogeneity in the evaluated sheep was not low. Results from the morphology suggest that Sudán and Etiope sheep should be classified as different breeds.
En el caso de los Ovinos de Pelo Criollos Colombianos (OPC), existen poblaciones que difieren considerablemente en sus características de fenotipo exterior, por tal motivo algunos autores como Bautista (1980), Bautista y Salazar (1980), Mason (1981), Peralta (1995), Ucrós (2001), Arcos et al. (2002) y Nigrinis (2013), consideran que existen dos tipos, razas o subrazas de mínimas o marcadas diferencias. Estas son la Sudán 
y la Etiope. Otros investigadores como Alfonso et al. (2000) y Vivas (2013), consideran que existe una tercera raza o subraza llamada Abisinio. Mientras que Roncallo et al. (1999), Gutiérrez et al. (2003), Vásquez et al. (2007), Carneiro (2008), Martínez et al. (2009), Montes et al. (2013), Moreno et al. (2013), Ocampo (2014) y Ángel y Ramírez (2014), no mencionan que entre los OPC haya alguna clasificación o subdivisión.

Los OPC se distribuyen principalmente en los siete departamentos de la región Caribe colombiana (Bautista, 1980) y son llamados Camuros, Pelonas o de múltiples formas según la zona, pero han sido renombrados como Ovinos de Pelo Criollos Colombianos, con la intención de connotar sentido de pertenencia en el apelativo, que además, se ha representado con la sigla propuesta por ASOOVINOS (Asociación de Criadores de Ganado Ovino de Colombia). Es de destacar, que dicha asociación está gestionando un adecuado reconocimiento racial de los OPC a nivel gubernamental, tomando como base el actual estudio.

Los productores y profesionales del área que acogen alguna de las diferidas perspectivas ya enunciadas sobre si hay una o más de una raza, al momento de intentar sustentarlo, llegan al punto de que sólo existe un trabajo que estudia comparativamente las distintas poblaciones de OPC, el cual está enfocado en la diversidad genética (Vivas, 2013). Mientras que este tipo de trabajos son inexistentes desde la morfología, dejando esto en evidencia que son pocas las investigaciones en cuanto a la etnología de este recurso zoogenético, temática que abarca el estudio morfoestructural de los animales. La carencia de estudios etnológicos en los OPC es preocupante, en especial porque estos se han visto amenazados en años recientes por el cruzamiento indiscriminado con razas foráneas, las cuales son mejoradas para la producción de carne, pero no para la adaptación al trópico bajo.

Para contrarrestar lo expuesto, es necesario el adecuado reconocimiento racial de los OPC, pues esta es la base para la conservación de una raza, el fomento de su uso sostenible y su mejora genética, acciones que pueden evitar su desaparición. Los recursos zoogenéticos, como los OPC, son valiosos debido a que están en la capacidad de albergar una diversidad genética que proporciona adaptación, la cual será de utilidad ante el cambio climático y la aparición de nuevas enfermedades (Rischkowsky y Pilling, 2010). Además, estos ovinos aparte de estar adaptados, igualmente se han destacado por ser mansos, prolíficos y proveedores de proteína animal, así como también han poseído una marcada importancia socio-cultural (Arcos et al., 2002). Debido a todas estas cualidades es prioritario reconocer adecuadamente a los OPC.

Por lo tanto, en el presente trabajo se evaluó morfoestructuralmente a hembras OPC Sudán y Etiope, para en base a ello determinar la organización morfoestructural de dichas poblaciones, y así comprobar la existencia de diferencias entre ellas, de tal manera que se contribuya a su reconocimiento como razas o subrazas diferenciadas.

\section{MATERIAL Y MÉTODOS}

\section{LOCALIZACIÓN DEL ESTUDIO Y RECOLECCIÓN DE DATOS}

Para realizar la evaluación morfoestructural de los ovinos Sudán (Figura 1) y Etiope (Figura 2), fueron seleccionadas hembras que cumplieran con la descripción clásica faneróptica y plástica del OPC realizada por Bautista (1980), quien manifiesta que los Sudán poseen un color de capa bayo, en algunos casos casi blanco, mientras que los Etiope se caracterizan por ser de una capa rojiza. Existe la nombradía de una posible tercera población de OPC llamado Abisinio, pero se imposibilitó su consecución en toda Colombia a través de múltiples medios y por consiguiente se descartó para este estudio. También, hay otro grupo que está compuesto por aquellos ovinos de pelo que no presentan uniformidad en sus características fenotípicas, pero que algunos los consideran criollos por encontrarse en sistemas de producción tradicionales. Sin embargo, Vivas (2013) en su investigación desde el campo de la genética, halló que los animales que evaluó con este tipo de características, eran resultado del cruzamiento de OPC con razas foráneas y por tal motivo no fueron considerados en esta investigación.

A lo largo de este trabajo se usará el término raza para hacer referencia a dos poblaciones con potencialidad de ser clasificadas como tal: Sudán y Etiope. Dentro de la raza Sudán los criadores hacían una distinción entre dos subpoblaciones con potencialidad de subrazas, a estas se les clasificaron como las subrazas Sudán Bayo (Figura 1A) y Sudán Blanco (Figura 1B), cuyo principal factor de diferenciación es el color de capa.

180 animales fueron evaluados, de los cuales 100 eran Sudán (80 Bayos y 20 Blancos) y 80 Etiope. La evaluación morfoestructural de los OPC se realizó en los municipios de Chimá, Ciénaga de Oro, Montería y Puerto Libertador del departamento de Córdoba; en el municipio de El Carmen de Bolívar, departamento Bolívar; en el municipio de Valledupar, departamento

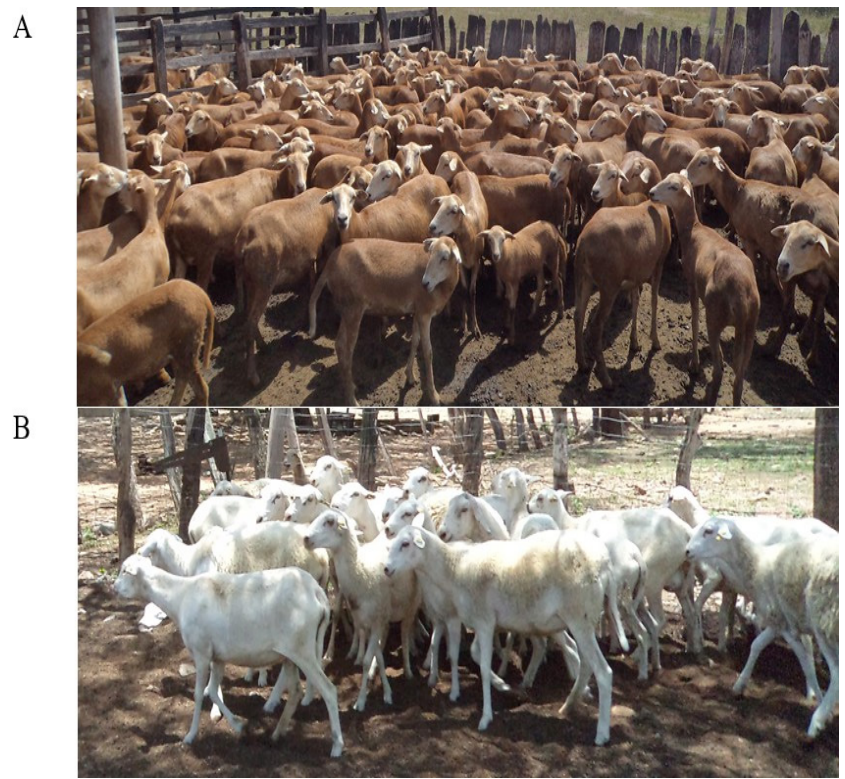

Figura 1. Ovejas Sudán (Sudán sheep). 


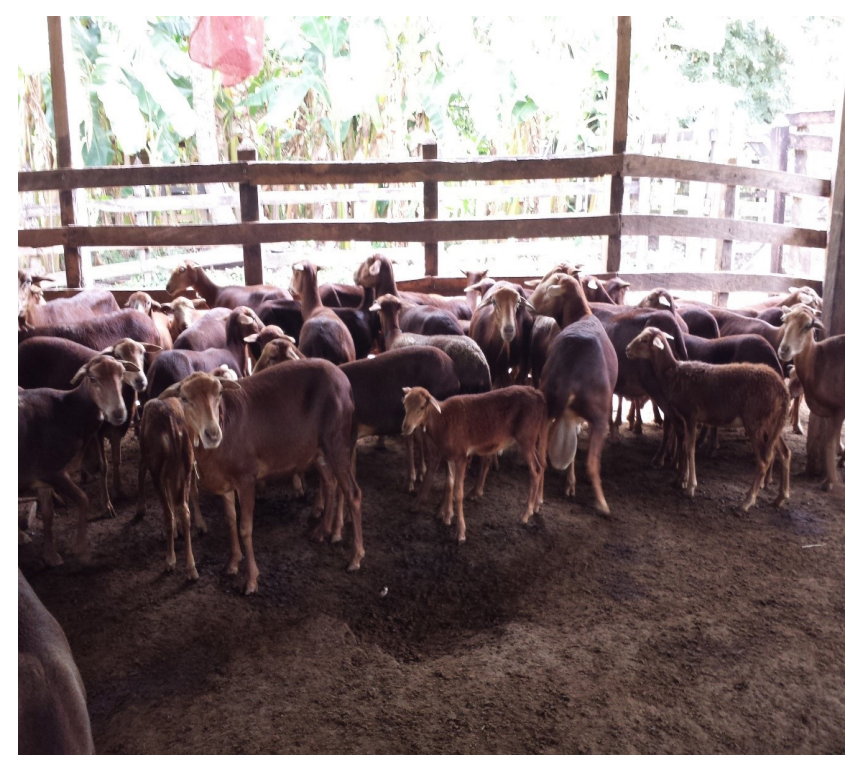

Figura 2. Ovejas Etiope (Etiope sheep).

Cesar; y en el municipio de San Juan del Cesar, departamento La Guajira. Las ovejas Sudán Bayo fueron muestreadas en Cesar y Córdoba, las Sudán Blanco en Cesar y La Guajira, y las Etiope en Córdoba y Bolívar. De acuerdo con el sistema de clasificación de las zonas de vida según Holdridge (2000), los municipios en los cuales se encontraban las Sudan Bayo pertenecen al Bosque Seco Tropical (Bs-T) y Bosque Muy Seco Tropical (Bms-T), las Sudán Blanco se encontraban en un medio catalogado como Bms-T, mientras que las Etiopes habitaban lugares que se consideran como Bs-T y Bosque Húmedo Tropical (Bh-T).

Las ovejas empleadas para este estudio fueron de una edad mayor o igual a los dos años, siendo calculada la edad a través de cronometría dentaria, previo a las mediciones hubo un periodo de ayuno de mínimo 12 horas y fue descartado cualquier ejemplar que presentara anomalías anatómicas, en el que se evidenciara cruzamiento con otra raza, preñez o enfermedad. Para la selección de las medidas zoométricas o variables morfoestructurales (Herrera y Luque, 2009), se tuvieron en cuenta los trabajos de Avellanet (2006), Parés (2008) y Parés (2009), quienes a su vez aplicaron la metodología zoométrica descrita por Aparicio (1960). Se escogieron un total de 17 variables, dentro de las cuales se incluyó el peso vivo (PV), ya que es un parámetro de interés productivo y altamente relacionado con las variables morfoestructurales.

Las variables evaluadas de acuerdo a la región se enuncian a continuación. Medidas cefálicas: longitud de la cabeza $(\mathrm{LCb})$, profundidad de la cabeza $(\mathrm{PCb})$, longitud del cráneo (LCn), longitud de la cara (LCr), ancho de cabeza $(\mathrm{ACb})$ y ancho del cráneo (ACn). Medidas del tronco: alzada a la cruz (ACr), alzada al esternón (AEs), alzada a la grupa (AG), longitud corporal (LCp), ancho entre encuentros (AEn), ancho entre iliones (AIl), ancho entre isquiones (AIq), longitud de la grupa (LG) y perímetro torácico (PT). Medidas de las extremidades: perímetro del metacarpo (PMc). Los instrumentos empleados en la evaluación de las variables estudiadas fueron: báscula portátil, bastón zoométrico, compás de espesores, cinta métrica y pie de rey.

\section{ANÁLISIS DE LOS DATOS}

Diversidad morfoestructural. A cada una de las variables estudiadas se les realizó estadística descriptiva: media, desviación estándar y coeficiente de variación $(\mathrm{CV})$. Además, para determinar si había diferencias $(\mathrm{p} \leq 0,05)$ entre las variables morfoestructurales según la raza y/o subraza de OPC, se realizó un análisis de varianza (ANOVA) y la prueba de Tukey-Kramer $(\mathrm{p} \leq 0,05)$. Posteriormente, se empleó un análisis de componentes principales, para distinguir cuáles medidas morfométricas y en qué proporción, fueron las causantes de la mayoría de la variación total entre las razas y subrazas de OPC. Luego, se efectuó un análisis discriminante canónico para determinar el porcentaje en que cada función canónica (CAN) explica la variabilidad entre los OPC, hallar las variables morfoestructurales con mayor potencia de discriminación y trazar una gráfica de la distribución canónica. Además, se realizaron las pruebas de Kolmogórov-Smirnov, Shapiro-Wilks, de esfericidad de Bartlett y la medición del parámetro Kaiser-Meyer-Olkin (KMO), para validar supuestos de normalidad y homogeneidad de varianzas.

Homogeneidad morfoestructural. Para determinar la homogeneidad de la morfoestructura dentro de cada raza y subraza, se realizó un test de homogeneidad a través de la estimación del CV de cada una de las variables morfoestructurales. Con el mismo fin, también fue evaluada la armonía del modelo morfoestructural, mediante la cuantificación del porcentaje de correlaciones positivas y significativas de los coeficientes de correlación de Pearson de las variables evaluadas.

Todos los procedimientos estadísticos fueron realizados a través de InfoStat ${ }^{\circledR}$ versión 2016I (Di Rienzo et al., 2016), a excepción de las pruebas KolmogórovSmirnov, Shapiro-Wilks, Bartlett y de la medición del parámetro KMO, las cuales fueron desarrolladas con el software SPSS (2010) versión 19,0.

\section{RESULTADOS Y DISCUSIÓN}

\section{DIVERSIDAD MORFOESTRUCTURAL}

Al observar los valores promedios, en términos generales se pudo notar que morfoestructuralmente las hembras Sudán presentaron cifras de mayor valor respecto a las Etiope (Tabla I). De acuerdo con el análisis de varianza, se encontró que las hembras Sudán Bayo y Sudán Blanco no presentaron diferencia estadísticamente significativa para el 70,59\% de las variables morfoestructurales evaluadas, resultados que reafirman el supuesto de semejanza manejado por los productores, en el cual se considera a los Sudán Bayo y a los Sudán Blanco como subrazas de la raza Sudán. En cambio, las hembras Sudán Bayo y Etiope presentaron diferencias estadísticamente significativas para el $88,23 \%$ de las medidas morfométricas, siendo un poco similar el caso de las Sudán Blanco frente a las Etiope, donde estas manifestaron diferencias estadísticamente significativas en el 64,7\% de las variables evaluadas. 
Tabla I. Estadísticos descriptivos y test de comparación de medias Tukey-Kramer $(\mathrm{P} \leq 0,05)$ en hembras OPC, para las variables morfoestructurales evaluadas de acuerdo con la raza y subraza (Descriptive statistics and comparison of means Tukey-Kramer $(\mathrm{P} \leq 0,05)$ in females OPC, for morphological traits evaluated according to the breed and sub-breed).

\begin{tabular}{|c|c|c|c|c|}
\hline Variables morfoestructurales $(\mathrm{cm})$ & & Etiope & Sudán Bayo & Sudán Blanco \\
\hline & Media & $36,01^{a}$ & $40,4^{b}$ & $40,16^{b}$ \\
\hline \multirow[t]{3}{*}{$\mathrm{PV}^{*}$} & DE & 6,58 & 5,64 & 8,68 \\
\hline & CV & 18,27 & 13,96 & 21,61 \\
\hline & Media & $18,41^{\mathrm{a}}$ & $20,34^{b}$ & $19,95^{b}$ \\
\hline \multirow[t]{3}{*}{$\mathrm{LCb}$} & $\mathrm{DE}$ & 2,51 & 1,03 & 0,91 \\
\hline & $\mathrm{cV}$ & 13,63 & 5,06 & 4,58 \\
\hline & Media & $14,16^{\mathrm{a}}$ & $14,57^{\mathrm{a}}$ & $14,18^{\mathrm{a}}$ \\
\hline \multirow[t]{3}{*}{$\mathrm{PCb}$} & $\mathrm{DE}$ & 1,44 & 0,72 & 0,92 \\
\hline & $\mathrm{cV}$ & 10,15 & 4,96 & 6,47 \\
\hline & Media & $10,77^{\mathrm{a}}$ & $12,03^{b}$ & $10,79^{a}$ \\
\hline \multirow[t]{3}{*}{ LCn } & DE & 1,44 & 1,03 & 0,97 \\
\hline & $\mathrm{CV}$ & 13,42 & 8,59 & 9,01 \\
\hline & Media & $14,6^{\mathrm{a}}$ & $15,92^{\mathrm{b}}$ & $15,5^{\mathrm{b}}$ \\
\hline \multirow[t]{3}{*}{$\mathrm{LCr}$} & DE & 1,27 & 0,99 & 0,99 \\
\hline & $\mathrm{CV}$ & 8,71 & 6,22 & 6,41 \\
\hline & Media & $11,29^{a}$ & $11,88^{b}$ & $11,42^{\mathrm{a}}$ \\
\hline \multirow[t]{3}{*}{$\mathrm{ACb}$} & DE & 0,61 & 1,09 & 0,7 \\
\hline & $\mathrm{CV}$ & 5,41 & 9,18 & 6,16 \\
\hline & Media & $8,27^{\mathrm{ab}}$ & $8,54^{b}$ & $8,15^{\mathrm{a}}$ \\
\hline \multirow[t]{3}{*}{$\mathrm{ACn}$} & $\mathrm{DE}$ & 0,72 & 0,34 & 0,38 \\
\hline & $\mathrm{CV}$ & 8,69 & 3,98 & 4,71 \\
\hline & Media & $62,14^{a}$ & $67,14^{b}$ & $67,59^{b}$ \\
\hline \multirow[t]{3}{*}{$\mathrm{ACr}$} & $\mathrm{DE}$ & 4,17 & 2,92 & 4,43 \\
\hline & $\mathrm{CV}$ & 6,71 & 4,36 & 6,55 \\
\hline & Media & $35,83^{a}$ & $41,16^{b}$ & $41,62^{b}$ \\
\hline \multirow[t]{3}{*}{ AEs } & $\mathrm{DE}$ & 3,5 & 3,67 & 3,24 \\
\hline & $\mathrm{CV}$ & 9,76 & 8,91 & 7,78 \\
\hline & Media & $63,88^{a}$ & $68,22^{b}$ & $68,52^{b}$ \\
\hline \multirow[t]{3}{*}{$A G$} & $\mathrm{DE}$ & 3,9 & 2,68 & 3,61 \\
\hline & CV & 6,1 & 3,92 & 5,27 \\
\hline & Media & $69,93^{a}$ & $74,63^{b}$ & $72,36^{b}$ \\
\hline \multirow[t]{3}{*}{ LCp } & $\mathrm{DE}$ & 4,57 & 4,18 & 5,02 \\
\hline & CV & 6,53 & 5,6 & 6,93 \\
\hline & Media & $13,82^{\mathrm{a}}$ & $14,77^{b}$ & $14,78^{b}$ \\
\hline \multirow[t]{3}{*}{ AEn } & $\mathrm{DE}$ & 1,6 & 1,32 & 2,19 \\
\hline & CV & 11,58 & 8,93 & 14,8 \\
\hline & Media & $14,04^{a}$ & $16,46^{b}$ & $15,27^{c}$ \\
\hline \multirow[t]{3}{*}{ All } & $\mathrm{DE}$ & 1,72 & 1,23 & 1,81 \\
\hline & CV & 12,22 & 7,46 & 11,83 \\
\hline & Media & $5,75^{a}$ & $6,22^{\mathrm{b}}$ & $6,4^{\mathrm{b}}$ \\
\hline \multirow[t]{3}{*}{ Alq } & $\mathrm{DE}$ & 0,94 & 0,82 & 0,67 \\
\hline & CV & 16,4 & 13,22 & 10,55 \\
\hline & Media & $19,47^{a}$ & $20,76^{b}$ & $20,39^{b}$ \\
\hline \multirow[t]{3}{*}{ LG } & $\mathrm{DE}$ & 1,48 & 1,3 & 1,65 \\
\hline & CV & 7,6 & 6,28 & 8,09 \\
\hline & Media & $78,58^{a}$ & $80,64^{a}$ & $80,34^{a}$ \\
\hline \multirow[t]{3}{*}{ PT } & $\mathrm{DE}$ & 6,53 & 4,26 & 9,09 \\
\hline & $\mathrm{CV}$ & 8,31 & 5,28 & 11,31 \\
\hline & Media & $6,87^{a}$ & $7,46^{\mathrm{b}}$ & $7,13^{\mathrm{a}}$ \\
\hline \multirow[t]{2}{*}{ PMc } & $\mathrm{DE}$ & 0,65 & 0,42 & 0,58 \\
\hline & CV & 9,41 & 5,6 & 8,16 \\
\hline
\end{tabular}

Medias con diferente letra son estadísticamente diferentes (P $\leq 0 ; 05)$; *unidad de medida en kilogramos; PV: peso vivo; LCb: longitud de la cabeza; PCb: profundidad de la cabeza; LCn: longitud del cráneo; LCr: longitud de la cara; ACb: ancho de cabeza; ACn: ancho del cráneo; ACr: alzada a la cruz; AEs: alzada al esternón; AG: alzada a la grupa; LCp: longitud corporal; AEn: ancho entre encuentros; All: ancho entre iliones; Alq: ancho entre isquiones; LG: Iongitud de la grupa; PT: perímetro torácico; PMc: perímetro del metacarpo; DE: desviación estándar; CV: coeficiente de variación en porcentaje (Means with different letters are

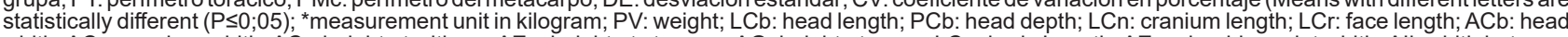
width; ACn: cranium width; ACr: height at withers; AEs: height at sternum; AG: height at rump; LCp: body Length; AEn: shoulder point width; All: width between ilia; Alq: width between ischia; LG: rump length; PT: chest girth; PMc: shin circumference; DE: standard deviation; CV: coefficient of variation in percentage). 
Según el análisis de componentes principales, se encontró que el $100 \%$ de la varianza total observada estaba explicada por dos componentes (CP), de los cuales el CP1 explicaba el 82,9\% de la variabilidad de los datos y el CP2 el 17,1\% de variabilidad restante. En cuanto a los autovectores obtenidos, todas las variables morfoestructurales estuvieron en un rango de 0,20 a 0,27 a excepción del ACn que tuvo un autovector de 0,14 , por lo que casi todas las variables presentaron una inercia o peso similar al momento de explicar la variabilidad de las observaciones. Se impide conformar un grupo de datos más pequeño que dé explicación a la variabilidad entre las razas estudiadas, porque la mayor parte de las variables morfoestructurales presentan una fuerte correlación entre ellas mismas. Estos resultados difieren a lo determinado por Moreno et al. (2013) en hembras OPC del departamento de Sucre, debido a que dichos autores obtuvieron un grupo de variables morfométricas con mayor poder de discriminación.

A través del análisis del biplot (Figura 3), se infiere que las subrazas de ovejas Sudán son relativamente cercanas entre sí, tal como se observa en el sector derecho del gráfico; dichas subrazas preservan una considerable distancia con respecto a las Etiope, ubicadas en el sector izquierdo del biplot. Esta figura es una representación del grado en el que influyen las variables morfoestructurales sobre la diferenciación de las OPC evaluadas. Además, se puede apreciar que algunas medidas zoométricas están solapadas, puesto a que tienen una capacidad similar de influir sobre la variabilidad de los parámetros evaluados.

Estos resultados difieren a lo encontrado por Arredondo et al. (2015) en ovejas Pelibuey, en el sentido que ellos requirieron de 12 CP para explicar el 100\% de la variabilidad de los datos; el caso de las ovejas criollas de Argentina es similar, donde se necesitaron de $13 \mathrm{CP}$ para explicar el $100 \%$ de la variabilidad de los datos (Peña et al., 2013).

Según el análisis discriminante canónico el 100\% de la variabilidad observada fue explicada por dos funciones canónicas estimadas, donde la CAN1 explicó el $84,81 \%$ y la CAN2 el $15,19 \%$ restante, teniendo ambas la capacidad de separar significativamente grupos de

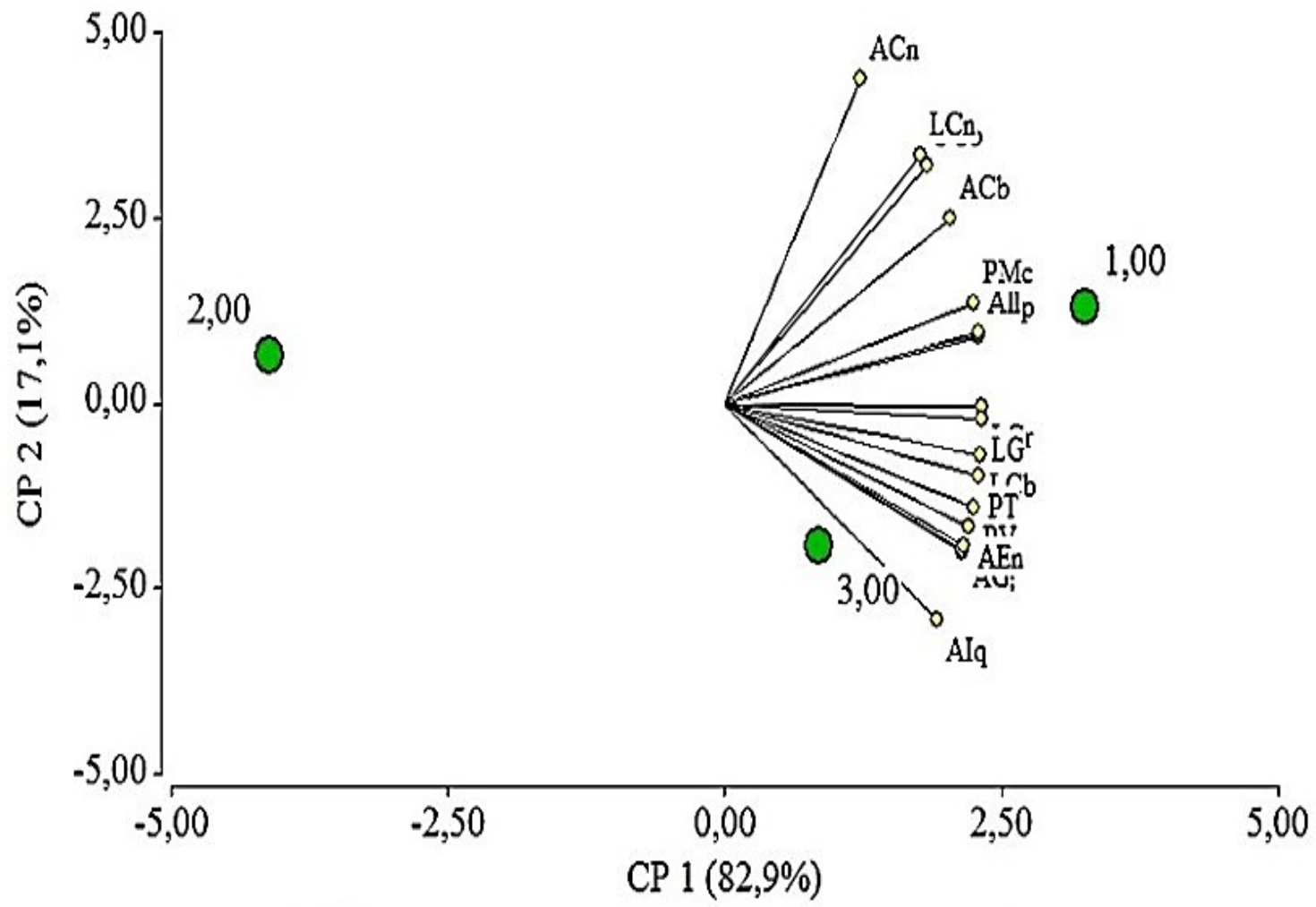

\section{O y 3 son Sudán Bayo y Blanco, 2 es Etiope -0 -Variables morfoestructurales}

O 1 and 3 are Sudán Bayo and Sudán Blanco, 2 is Etiope
-0 Morphological traits

Figura 3. Biplot del análisis de componentes principales de las variables morfoestructurales de las OPC Sudán y Etiope (Biplot of principal component analysis of the morphological traits of OPC Sudán and Etiope). 
acuerdo al test Lambda de Wilks ( $p \leq 0,01)$. La CAN1 está dominada por la alta carga positiva del AIl y la LCn, y la alta carga negativa del PT y el PV, mientras que la CAN2 está determinada por la alta carga positiva del ACn y la alta carga negativa del AIq y la ACr. En el diagrama de dispersión presentado en la Figura 4, se aprecia que, aunque existe la tendencia de formarse poblaciones separadas, hay cierto grado de conexión entre las razas evaluadas, lo que puede estar explicado por el origen en común que poseen los OPC.

En una investigación hecha por Traoré et al. (2008), se estudiaron tres razas de ovejas (Burkina-Sahel, Djallonké y Mossi) en tres distintas áreas de Burkina Faso, hallándose que la CAN1 y la CAN2 explicaban el 90,9 y $9,1 \%$ de la variación total, respectivamente, siendo ambas CAN estadísticamente significativas $(\mathrm{p} \leq 0,01)$. Por lo que, ambas ayudan significativamente a la discriminación entre los grupos de dicho estudio. Por su parte, Asamoah-Boaheng y Sam (2016), trabajaron con varios cruces ovinos (Sahell/West African Long Legged, Djallonke/West African Dwarfs y razas cruzadas/híbridos), donde la CAN1 explicó el 93,1\% de la variación y la CAN2 explicó sólo el 6,9\%, teniendo la CAN1 significancia $(\mathrm{p} \leq 0,01)$ en la discriminación de los grupos, mientras que la CAN2 no. Diaz et al. (2003) examinaron la varianza total en las razas ovinas Pomarancina, Garfagnina Bianca, Appenninica y Massese, y encontraron que tres CAN explicaron el 72,71, 16,43 y $10,95 \%$ de la variación.

Las ovejas Etiope presentan un menor potencial anatómico-estructural respecto a las Sudán, debido a la responsabilidad de varios factores, entre ellos las diferencias entre dichas razas de OPC, que según lo que se demostró son significativas. Otros factores, que podrían tener un mayor efecto, son los medioambientales, como el nicho ecológico en el que se desenvolvieron cada una de estas poblaciones, pues a pesar de que se encuentran en el trópico bajo colombiano, más específicamente en la región Caribe, los OPC se esparcen desde ecosistemas de Maleza Desértica (Md-T) hasta de Bh-T (Holdridge, 2000), condiciones agroecológicas que presentan diferencias y que de distinta manera influyen en la conformación morfoestructural de los ovinos. Esto se puede respaldar por estudios como los de Sun et al. (2009), quienes hallaron divergencias entre poblaciones ovinas que se distribuían en distintos tipos de macrohábitats. Además, Wainwright y Reilly (1994) exponen que es posible que se dé la existencia de fuertes correlaciones entre el tipo de hábitat y la morfología, independiente de la relación filogenética de los animales.

Con base en lo anteriormente expuesto, no es errado o falto de fundamento, considerar que las poblaciones Sudán y Etiope por aislamiento e influencias del medio se han logrado distanciar genéticamente (Vivas, 2013), y que esto haya conllevado a la variación de su morfoestructura, hasta el punto de que se puedan considerar como distintas racialmente. Por otra parte, Rege (2001), mediante su investigación indica que fenotipos distintos son la consecuencia de eventos de aislamiento geográfico de los animales, cosa que es deducible para los OPC de acuerdo con los hallazgos morfoestructurales que han sido relatados; esta es una noción que está acorde con las consideraciones entre medio y raza que hace Laurans (1982).

\section{HOMOGENEIDAD MORFOESTRUCTURAL}

En una población animal, se puede usar el test de homogeneidad morfoestructural como una prueba

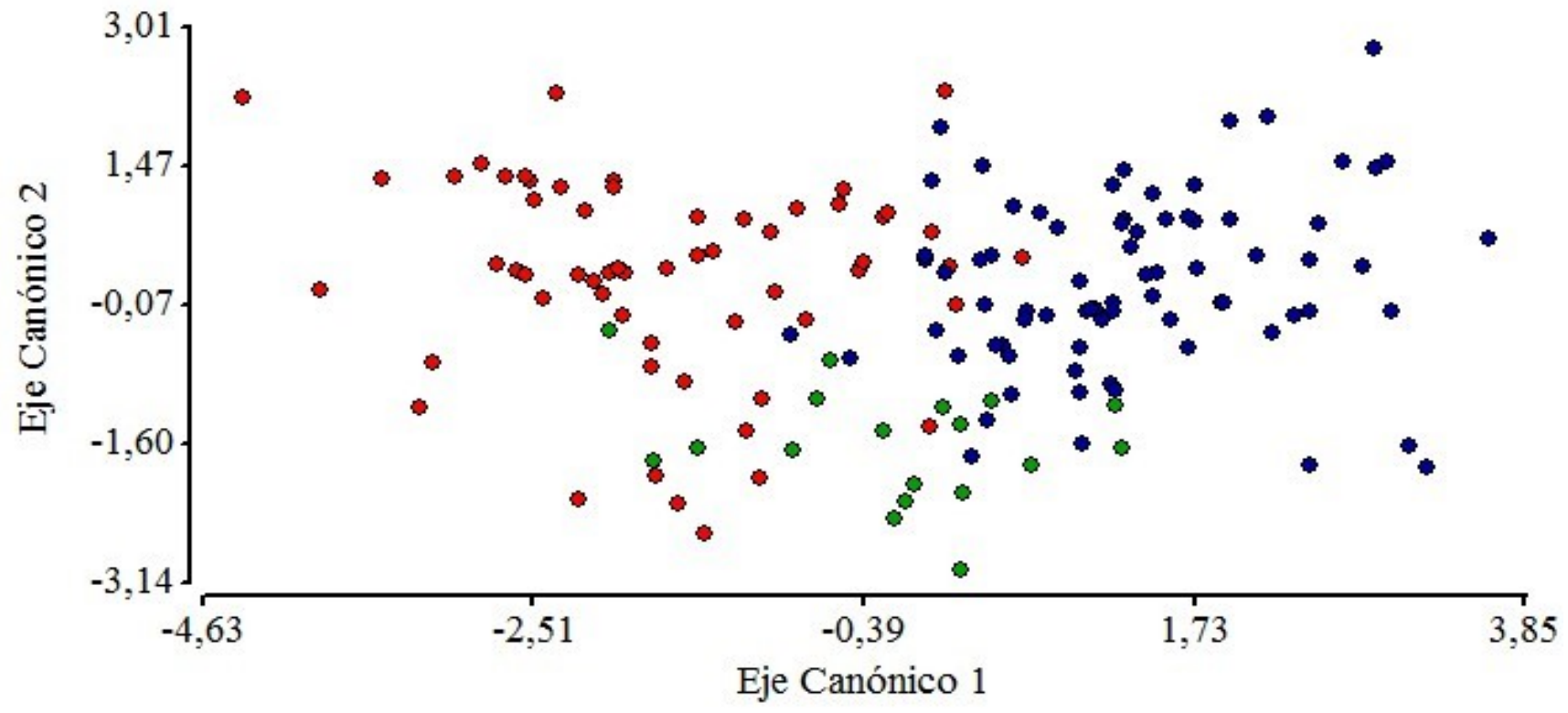

Sudán Blanco

Etiope

Sudán Bayo

Figura 4. Representación canónica de las variables morfoestructurales asociadas con los OPC de acuerdo con la raza y subraza (Canonical representation of morphological traits associated with OPC according to the breed and sub-breed). 
para establecer el grado de variación de la morfoestructura, dicho test se realiza a través del cálculo del $\mathrm{CV}$, para su interpretación se debe tener en cuenta que los porcentajes más altos representan una mayor heterogeneidad, mientras que los más bajos una mayor homogeneidad (Roche et al., 2006; Herrera y Luque, 2009). Mucha variabilidad puede deberse a introgresiones o a adaptaciones a diferentes sistemas de cría dentro de una población.

En las variables de la región cefálica, se observó que las ovejas Sudán Bayo y Sudán Blanco tuvieron CV que oscilaron entre el 3,98 y el 9,18\% y del 4,58 al $9,01 \%$, respectivamente, por lo que se puede decir que las Sudán poseen un grado de alto a mediano-alto de homogeneidad. En cuanto a la raza Etiope, esta tuvo un CV que fluctuó entre el 5,41 y 13,63\%, lo cual señala que las Etiope tienen un grado de homogeneidad mediano-alto tendiente a mediano. Datos de homogeneidades similares a las de ambas razas se hallaron en ovejas Pelibuey (Arredondo et al., 2013), las cuales presentaron CV que oscilaron entre un 6,23 y 10,78\%, mientras que valores de menor homogeneidad en ovejas OPC fueron reportados por Moreno et al. (2013), con CV que alcanzaron hasta un $24 \%$.

Respecto a las alzadas, se apreció que estas poseen un CV que osciló entre el 3,92 y 9,76\% para las dos razas, lo que quiere decir que presentan una homogeneidad alta tendiente a mediana-alta. Grados de homogeneidad similares para las alzadas han sido obtenidas en OPC por Moreno et al. (2013), por Arredondo et al. (2013) en la ACr de ovejas Pelibuey y por Álvarez et al. (2000) en la ACr de ovejas Canaria, con porcentajes que variaron del 5,98 al 8,2\%. Entrando a detallar las homogeneidades para el resto de las variables morfoestructurales del tronco en forma general, se aprecia que las OPC presentaron un grado de homogeneidad mediano-alto (del 5,28 al 16,4\%). CV similares a los del presente estudio para el PT, han sido reportados en OPC por Ángel y Ramírez (2014) y por Moreno et al. (2013), con valores de 6,8 y $8,7 \%$, respectivamente. Mientras que cifras obtenidas en otras investigaciones concernientes a las variables morfoestructurales de la grupa, están entre los porcentajes encontrados en el actual trabajo, hallándose valores de 9,7\% en hembras OPC del departamento de Sucre (Moreno et al., 2013), y de 9,13 a 9,18\% en ovejas Pelibuey (Arredondo et al., 2013).

Los CV del PMc oscilaron entre el 5,6 y 9,41\%, lo que indica que las hembras OPC de este estudio fueron de homogeneidad mediana-alta para esta variable, encontrándose coeficientes superiores, pero no muy distanciados, en ovejas OPC (Moreno et al., 2013) y en ovejas criollas del oeste formoseño (De la Rosa et al., 2012), con CV de 10,50 y 11,18\%, respectivamente.

Respecto a la armonía del modelo morfoestructural, esta se fundamenta en lo expuesto por Lerner y Donald (1969), quienes explican que la mayor parte de los genes que influyen en la conformación morfoestructural de un animal no son de acción local, sino general. Por lo tanto, mediante la estimación del porcentaje de correlaciones positivas y significativas $(p \leq 0,05)$, se comprueba la existencia de armonía en el modelo mor- foestructural, ya que entre más alto sea el porcentaje de dichas correlaciones, quiere decir que mayor es el grado de armonía en una población.

El porcentaje de correlaciones positivas y estadísticamente significativas en las OPC fueron superiores al $50 \%$, teniendo las Sudán Bayo y las Sudán Blanco un valor de 58,82\% cada una, y las Etiope un $62,28 \%$ de correlaciones positivas y significativas, lo que se traduce en que ambas razas son de una mediana armonía en su modelo morfoestructural, es decir, que las Etiope se asemejan entre ellas, que las Sudán Bayo son similares entre sí y que para las Sudán Blanco también aplica este mismo análisis. Resultados de armonía mediana, al igual que los de este estudio, fueron reportados en ovejas de la raza Ansotana, con un 51,47\% de correlaciones positivas y estadísticamente significativas (Roche et al., 2010), mientras que armonía mediana-baja en el modelo morfoestructural ha sido reportada en ovejas de la raza Cartera, con un 38,97\% de correlaciones positivas y significativas (Lara et al., 2004), también, se han reportado armonías por debajo del anterior valor en la raza ovina Ojinegra, la que obtuvo un porcentaje de 22,22\% (Picazo et al., 2004), grado de armonía del modelo morfoestructural que es catalogado como bajo.

Lo mencionado con anterioridad, permite ver que el grado de armonía de las variables morfoestructurales hallada en cada raza y subraza, es semejante e incluso superior a lo encontrado en algunas poblaciones ovinas con reconocimiento racial. Estos resultados normalmente no son de esperar en poblaciones criollas, pero algunos casos pueden estar influidos por carencia de manejos reproductivos que posiblemente favorecieron a la consanguinidad.

\section{CONCLUSIONES}

A través de los procedimientos estadísticos utilizados, se pudo determinar que las razas de OPC evaluadas presentan un considerable grado de diversidad intergrupalmente, pero que a pesar de ello, existe poca heterogeneidad a nivel intragrupal; estos resultados permiten sugerir desde la morfología cuantitativa, que los Sudán y Etiope sean catalogados como diferentes racialmente. Se responsabiliza de la variabilidad existente, a la exposición que los OPC tuvieron generaciones atrás a escenarios ambientales diferentes, los cuales permitieron que se generara un efecto del medio sobre la morfoestructura que en cada población se fue fijando genéticamente.

Considerando el amplio debate existente en torno a qué es una raza, los autores concluyen que la interpretación apropiada de lo ya expuesto radica en comprender que los Sudán y Etiope son dos poblaciones diferentes, que se considerarán como razas diferentes o como una misma raza con varias subrazas, no sólo en base a lo evaluado desde lo biológico, sino, teniendo en cuenta también la interacción de ello con elementos culturales y políticos, pero, lo fundamental es tener consciencia de que no está bien que no exista discriminación entre estas poblaciones.

\section{AGRADECIMIENTOS}


Los autores agradecen a Maximiliano Ambrosio, vicepresidente de ASOOVINOS, por facilitar los contactos con las producciones muestreadas, a Mario Cristino Ibargüen Asprilla, estudiante de Medicina Veterinaria y Zootecnia de la Universidad de Córdoba, por la colaboración prestada en la recolección de datos, y a la Universidad de Córdoba por el incentivo económico brindado. La presente investigación tuvo apoyo financiero de la Universidad de Córdoba, a través de la Convocatoria Interna de Investigación en Programas de Pregrado (Acuerdo 014 de 2015).

\section{BIBLIOGRAFÍA}

Alfonso, A, Bahamón, A \& Grajales, L 2000, ‘Análisis del Desempeño Productivo y Reproductivo de un Rebaño de Ovejas Africanas en la Zona del Valle del Cauca-Trópico Bajo Colombiano', Revista de Medicina Veterinaria y de Zootecnia, vol. 47, no. 2, pp. 7-10.

Álvarez, S, Fresno, M, Capote, J, Delgado, J \& Barba, C 2000, 'Estudio para la caracterización de la raza ovina Canaria', Archivos de Zootecnia, vol. 49, no. 185, pp. 209-215.

Ángel, S \& Ramírez, A 2014, ‘Estudio zoométrico del ovino de pelo criollo colombiano de la zona norte del departamento del Huila (Colombia)', Actas Iberoamericanas de Conservación Animal, vol. 4, pp. 338-340.

Arcos, J, Romero, H, Vanegas, M\& Riveros E 2002, Ovinos Colombianos de Pelo, CORPOICA, Tolima.

Arredondo, V, Macedo, R, Magaña, J, Molina, J, Prado, O, García, L, Lee, H \& Herrera, A 2013, 'Variabilidad morfológica de la oveja Pelibuey en colima, México', Actas Iberoamericanas de Conservación Animal, vol. 3, pp. 1-7.

Arredondo, V, Macedo, R, Molina, J, Magaña, J, Prado, O \& García, L 2015, ‘Análisis multivariado de la variación morfológica de la oveja Pelibuey en Colima, México, 'Actas Iberoamericanas de Conservación Animal, vol. 5, 87-92.

Asamoah-Boaheng, M \& Sam, E 2016, 'Morphological characterization of breeds of sheep: a discriminant analysis approach', SpringerPlus, vol. 5 no. 69 , visto el 5 de noviembre de 2016, https://www.ncbi. nlm.nih.gov/pmc/articles/PMC4723378/.

Avellanet, R 2006, 'Conservación de recursos Ovinos en la raza Xisqueta: Caracterización estructural, Racial y Gestión de la diversidad en programas "In situ"' tesis PhD, Universitat Autònoma de Barcelona, visto el 5 de noviembre de 2016, https://ddd.uab.cat/pub/tesis/2006/ tdx-0314107-163422/ratlde 1.pdf.

Bautista, R 1980, 'Apuntes Sobre Ovino Africano en Colombia', Carta Agraria, pp. 17-19.

Bautista, R \& Salazar, J 1980, Ovinos Prolíficos Tropicales, FAO y PNUMA, Roma.

Carneiro, H 2008, 'Caracterização morfológica de ovinos no Brasil, Uruguai e Colômbia' trabajo de grado de maestría, Universidade de Brasilia, visto el 5 de noviembre de 2016, http://repositorio.unb.br/ handle/10482/3009.

DAD-IS, 2016, Domestic Animal Diversity Information System, visto el 5 de noviembre de 2016,http://www.fao.org/dadis/.

De la Rosa, S, Revidatti, M, Tejerina, E, Orga, A, Cappello, J \& Petrina, J2012, 'Estudio para la caracterización de la oveja criolla en la región semiárida de Formosa, Argentina', Actas Iberoamericanas de Conservación Animal, vol. 2, pp. 87-94.

Delgado, J, León, J, Gómez, M, Nogales, S \& Camacho, M 2009, 'Las Razas Ovinas Ibéricas y su Participación en la Colonización de lberoamérica', en: J Delgado \& S Nogales (eds.), Biodiversidad Ovina Iberoamericana. Caracterización y Uso Sustentable, Córdoba, pp. 17-30, visto el 5 de noviembre de 2016, http://www.uco.es/ conbiand/pdf/biodiversidad_ovina.pdf.

Di Rienzo, J, Balzarini, M, Casanoves, F, González, L, Tablada, M\& Robledo, C 2016, InfoStat: software estadístico, Universidad Nacional de Córdoba, Córdoba.
Diaz, P, Degl'Innocenti, P, Sargentini, C, Moretti, M \& Bozzi, R 2003, 'Phenotypic diversity of Tuscany's endangered sheep breeds: A canonical discriminant approach', Italian Journal of Animal Science, vol. 2 no. 1, pp. 563-565.

Gutiérrez, W, Martínez, R, Escobedo, C \& Anzola, H 2003, Situación de los Recursos Zoogenéticos en Colombia, Ministerio de Agricultura y Desarrollo Rural, Bogotá.

Herrera, M \& Luque, M 2009, 'Morfoestructura y Sistemas para el Futuro en la Valoración Morfológica', en: C Sañudo (ed.), Valoración Morfológica de los Animales Domésticos, Madrid, Ministerio de Medio Ambiente y Medio Rural y Marino, pp. 83-101, visto el 5 de noviembre de 2016,http://www.mapama.gob.es/es/ganaderia/ temas/zootecnia/razas-ganaderas/publicaciones-interes/LIBRO_ valoracion_morfologica_SEZ_tcm7-306042.pdf.

Holdridge, L 2000, Ecología Basada en Zonas de Vida, Editorial IICA, San José.

Lara, S, Picazo, R, Fuentes, P, Peña, F \& Herrera, M 2004, 'Raza ovina Cartera. 1. Caracteres cuantitativos morfoestructurales', en: XXIX Jornadas de la Sociedad Española de Ovinotecnia y Caprinotecnia, Imprenta Diputació de Lleida, Lleida, visto el 5 de noviembre de 2016,http://seoc.eu/es/2004-lleida/.

Laurans, R 1982, 'L'évolution du concept de race en zootechnie', Ethnozootechnie, vol. 29, pp. 5-6.

Lerner, I \& Donald, H 1969, La nueva Zootecnia, Ed. Academia León, Sevilla.

Martínez, R, Vásquez, R \& Ballesteros, H 2009, 'El ovino criollo en Colombia, conservación, caracterización y evaluación de la variabilidad genética', en: J Delgado \& S Nogales (eds.), Biodiversidad Ovina Iberoamericana. Caracterización y Uso Sustentable, Córdoba, pp. 17-30, visto el 5 de noviembre de 2016, http://www.uco.es/ conbiand/pdf/biodiversidad_ovina.pdf.

Mason, I 1981, 'Razas Indígenas de Ovinos y Caprinos en América Latina', en: B Müller-Haye \& J Gelman (eds.), Recursos Genéticos Animales en América Latina, FAO, Italia, pp. 132-137.

Montes, D, Moreno, J, Hurtado-Lugo, N, Ramírez, R, Celis, A \& Garay, G 2013, 'Caracterización faneróptica y morfológica de la hembra ovina de pelo criollo (Camura) colombiana, en la Sub Región Sabanas y Golfo de Morrosquillo departamento de Sucre', Revista Colombiana de Ciencia Animal, vol. 5, no. 1, pp. 104-115.

Moreno, J, Montes, D, Ucrós, J, Fernández, A \& Cardona, J 2013, 'Variabilidad morfoestructural de la hembra ovina de pelo criollo colombiana', Livestock Research for Rural Development, vol. 25, no. 5, visto el 5 de noviembre de 2016,http://www.lrrd.org//rrd25/5/ more25083.htm.

Nigrinis, G 2013, Los Ovinos Tropicales de Pelo, Más de 500 Años de Selección Natural, Imprenta Mercantil de los Hermanos Nigrinis, Girón.

Ocampo, R 2014, 'Caracterización genética de ovinos en Colombia por medio de marcadores microsatélites', trabajo de grado de Maestría, Universidad de Antioquia, visto el 5 de noviembre de 2016, http:// bibliotecadigital.udea.edu.co/handle/10495/1902.

Parés, P 2008, 'Caracterització estructural i racial de la raça ovina aranesa' tesis $\mathrm{PhD}$, Universitat Autònoma de Barcelona, visto el 5 de noviembre de 2016, http://www.tdx.cat/bitstream/handle/10803/5709/ pmpc l de 1 . pdf; isessionid=6292E3CDFA 11 438F4F701966A5D0 C3D5? sequence $=1$.

Parés, P 2009, 'Zoometría. Importancia de la Morfología y su Valoración en los Animales Domésticos', en: C Sañudo (ed.), Valoración Morfológica de los Animales Domésticos, Ministerio de Medio Ambiente y Medio Rural y Marino, Madrid, pp. 23-46, visto el 5 de noviembre de 2016,http://www.mapama.gob.es/es/ganaderia/temas/ zootecnia/razas-ganaderas/publicaciones-interes/LIBRO_valoracion_morfologica_SEZ_tcm7-306042.pdf.

Peña, S, López, G, Martínez, R, Abbiati, N, Castagnasso, E, Giovambattista, G \& Genero, E 2013, 'Características zoométrica de ovinos criollos de cuatro regiones de la Argentina', Actas Iberoamericanas de Conservación Animal, vol. 3, pp. 174-181. 
Peralta, E 1995, Antecedentes de los Ovinos de Pelo en Colombia, en: Memorias Ovinos Colombianos de Pelo, Curso Sobre Producción de Ovinos Colombianos de Pelo, CORPOICA, Ibagué, pp. 8-14.

Picazo, R, Lara, S, Fuentes, P, González, A \& Herrera, M 2004, ’Raza ovina Ojinegra: caracteres cuantitativos morfoestructurales', en: XXIX Jornadas de la Sociedad Española de Ovinotecnia y Caprinotecnia, Imprenta Diputació de Lleida, Lleida, visto el 5 de noviembre de 2016, http://seoc.eu/es/2004-lleida/.

Rischkowsky, B \& Pilling, D (eds.) 2010, La Situación de los Recursos Zoogenéticos Mundiales para la Alimentación y la Agricultura. Food and Agriculture Organization of the United Nations (FAO), Roma, visto el 12 de abril de 2018, http://www.fao.org/docrep/012/ al250s/al250s.pdf.

Rege, E 2001, 'Defining livestock breeds in the context of communitybased management of farm animal genetic resources', en: D Vilakati, C Morupisi, L Setshwaelo, C Wollny, A von Lossau \& A Drews (eds.), Community-based management of animal genetic resources. FAO, Swaziland, pp. 27-35.

Roche, A, Boscolo, J, González, A \& Herrera, M 2010, 'Raza ovina Ansotana I. Caracteres cuantitativos morfoestructurales', en: XXXV Congreso de la Sociedad Española de Ovinotecnia y Caprinotecnia, Gráficas Germinal S.C.L., Valladolid, visto el 5 de noviembre de 2016, http://seoc.eu/es/2010-valladolid/.

Roche, A, Salinas, M, Santander, L, Andaluz, E, Peña, F \& Herrera, M 2006, 'Raza ovina Roya Bilbilitana. Caracteres cuantitativos Morfoestructurales', en: XXI Jornadas Científicas y X internacionales de la Sociedad Española de Ovinotecnia y Caprinotecnia, Gráficas Germinal S.C.L., Zamora, visto el 5 de noviembre de 2016,http:// seoc.eu/es/2006-zamora/.

Roncallo, B, Toloza, A, Barros, J, Silva, J, Araujo, A, Mejía, M, Ávila, E\& Robledo, L 1999, Sistemas de producción ovino-caprinos en los departamentos de La Guajira, Cesar y Magdalena, CORPOICA, Valledupar.

SPSS, 2010, Computer Software 19,0 SPSS inc., Chicago.

Sun, W, Chang, H, Musa, H, Yang, Z, Tsunoda, K, Ren, Z \& Geng, R 2009, 'Influence of environmental factors on the genetic diversity of sheep', Journal of Animal and Veterinary Advances, vol. 8, no. 6, pp. 1070-1074.

Traoré, A, Tamboura, H, Kaboré, A, Royo, L, Fernández, I, Álvarez, I, Sangaré, M, Bouchel, D, Poivey, J \& Francois, D 2008, 'Multivariate characterization of morphological traits in Burkina Faso sheep', Small Ruminant Research, vol. 80, no. 1, pp. 62-67.

Ucrós, J 2001, 'Establecimiento de Parámetros Productivos y Ovinométricos en Ovinos Africanos Bajo Pastoreo en la Subregión Sabana de Sucre' trabajo de grado de pregrado, Universidad de Sucre, vista el 5 de noviembre de 2016, http://repositorio.unisucre.edu.co/ handle/001/501.

Vásquez, R, Martínez, R, Rodríguez, O, Ballesteros, H, Rodríguez, G, Gamarra, C \& Jiménez, F 2007, Manual para el Manejo de las Razas Criollas Ovino de Pelo y Caprino Dentro del Plan de Fomento, CORPOICA, Mosquera.

Vivas, N 2013, 'Diversidad Genética de Ovinos Criollos Colombianos' trabajo de grado de maestría, Universidad Nacional de Colombia Sede Palmira, visto el 5 de noviembre de 2016, http://www.bdigital. unal.edu.co/12683/1/7410001.2013.pdf.

Wainwright, P \& Reilly, S 1994, Ecological Morphology, University of Chicago Press, Chicago. 